Research Article

\title{
Heat Transfer Studies of Arrays of Prolate Particles in Gas-Solid Flows
}

\author{
Romana Basit $\mathbb{D}^{1},{ }^{1}$ Xinyang Li $\mathbb{D}^{1},{ }^{1}$ Zheqing Huang $\mathbb{D}^{1},{ }^{1}$ and Qiang Zhou $\mathbb{D}^{1,2}$ \\ ${ }^{1}$ School of Chemical Engineering and Technology, Xi'an Jiaotong University, Xi'an 710049, China \\ ${ }^{2}$ State Key Laboratory of Multiphase Flow in Power Engineering, Xi'an Jiaotong University, Xi'an 710049, China
}

Correspondence should be addressed to Qiang Zhou; zhou.590@mail.xjtu.edu.cn

Received 9 October 2020; Revised 25 October 2020; Accepted 26 October 2020; Published 12 November 2020

Academic Editor: Muhammad Mubashir Bhatti

Copyright ( $\odot 2020$ Romana Basit et al. This is an open access article distributed under the Creative Commons Attribution License, which permits unrestricted use, distribution, and reproduction in any medium, provided the original work is properly cited.

Numerical study of forced convection heat transfer from arrays of prolate particles is performed using the second-order Immersed Boundary-Lattice Boltzmann Method (IB-LBM). Prolate particle is studied with aspect ratio of 2.5 with solid volume fraction variation from 0.1 to 0.3 . For each solid volume fraction, arrays of prolate particles are generated and simulations have been performed to calculate Nusselt number for four different Hermans orientation factors and various Reynolds numbers. From the simulation results, it has been observed that, for any specific value of Hermans orientation factor, Nusselt number increases with the increase of the Reynolds number and solid volume fraction. More importantly, it is found that the effect of orientations on Nusselt number is significant. Nusselt number correlation is developed for ellipsoidal particles as function of Reynolds number, Prandtl number, solid volume fraction, and orientation factors. This correlation is valid for $0.1 \leq c \leq 0.3$ and $0<\operatorname{Re} \leq 100$.

\section{Introduction}

Fluid flow and its interaction with solid particles is an essential phenomenon, which has immense applications in the domain of various industries, for example, in pneumatic conveying system, drying of food items, combustion of coal, fluidized bed, waste recycling, and pharmaceutical product formation. Heat transfer of these gas-solid flows is a significant phenomenon that needs to be investigated. A comprehensive understanding of this process is essential for the better operations of equipment in which gas-solid flows take place.

Many studies on the heat transfer of multiparticle systems have been carried out; however, most of the research is done on spherical particles only. In practical engineering applications, most of the particles are nonspherical in shape and form diverse orientations in space. Ellipsoidal particles have many applications in industry, for example, in bio gas, in manufacturing industry, and in char conversion industry for energy conversion process [1]. These industries deal with particles having increasingly stretched shapes more like the prolate. Fuel used in the biomass procedure takes the shape of prolate particles [2]. Also, particles present in biomass process acquire different orientations. However, detailed analysis of many particle systems of prolate particles with different orientations has not been performed yet. In the present work, these orientations are quantified by using the Hermans orientation factors $(S)$ in the range of -0.5 to 1 . In the literature, dependence of Nusselt number $(\mathrm{Nu})$ is only presented in the form of solid volume fraction and Reynolds number. But, in the present research, a new parameter of $S$ is found, which has strong effects on the Nu. The present research aims to study such systems of particles; therefore, it will be an important contribution in the field of gas-solid flows.

Normally people use Gunn's [3] and Wakao et al.'s [4] correlations for predicting the heat transfer and drag in fluid particle systems which fit well only for spherical particle. Many researchers have investigated the heat transfer properties in the arrays of spherical particles [5-8]. Some investigators have performed numerical simulations to study the heat transfer from single nonspheroidal particles. Finite difference numerical procedure was used by Juncu for unsteady heat transfer studies of a prolate and an oblate 
particle [9]. The particle temperature was considered only to be a function of time, whereas it was considered uniform in space. In the simulations, Reynolds number range was taken from 10 to 200 and Prandtl number was chosen to be 1 and 10 with axis ratio from 0.1 to 0.9 . Richter and Nikrityuk performed numerical simulations in three dimensions for calculating heat transfer and drag force coefficients [10]. They used different shapes including ellipsoidal, spherical, and cuboidal particles in the flow, with Reynolds number in the range from 10 to 250 . In the study, they explored that the drag coefficient of a specific particle depended on its normalized longitudinal length, whereas Nusselt number was mostly affected by the crosswise sphericity and sphericity itself. Zhang et al. performed direct numerical simulation (DNS) studies of forced convection from rotating single ellipsoidal particles for five different aspect ratios [11]. They investigated the effect of particle rotations on drag, lift, and moment coefficients. Average value of Nusselt number was also analyzed. They concluded that particle rotations played a substantial role in momentum and heat transfer from ellipsoidal particles.

Yang et al. studied the forced convection heat transfer in packed bed reactors using CFX10 [12]. They employed the SC, BCC, and FCC structured packing of solids for heat transfer studies. They concluded that selection of structured packing affected the pressure drop in the packed bed reactors. They also studied the effect of the shape of particles on heat transfer and flow process. Their work was mainly for higher Reynolds number flows for which RNG $k-\varepsilon$ turbulence model was used. Tavassoli et al. used the DNS for heat transfer studies of nonspherical particles with fixed random arrays [13]. The main objective of the study was to modify the existing correlations of spherical particles to be used for nonspherical objects. Spherocylindrical particles with random distribution were used and the simulations were performed using the immersed boundary method (IBM). Simulation results showed that, by making proper selection of effective diameter, the correlations of spherical particles could be employed for spherocylindrical shape without introducing any significant errors. He and Tafti investigated the heat transfer from ellipsoidal particles for low-to-moderate Reynolds numbers in the range of 10 to 200 [14]. They used a particle aspect ratio of 2.5 with solid volume fraction in the range of 0.1 to 0.35 . Random assemblies of particles were generated by using SDK-PhysX. They reported a Nusselt number correlation based on the simulation data. Li et al. studied the effects of spheroid orientations on drag force of prolate particles by using IBM [15]. In particular, they adopted Hermans orientation factor (denoted by $S$ ) to quantify the mean orientation of the prolate. By definition, $S$ ranges from -0.5 to 1 and can be used to represent the mean orientation of all possible arrangements of ellipsoids. Different values of $S$ represent arrays of particles with different mean orientations (see Figure 1). Results obtained by Li et al. showed that drag force decreased with the rise of $S$ when flow followed the reference direction. A new drag correlation based on particle orientation, aspect ratio, and solid volume fraction was proposed for ellipsoidal particles.
In the literature, the available investigations of heat transfer phenomenon between prolate particles and the fluid are mostly focusing on an isolated particle or arrays of particles with random orientations. However, practical applications usually involve millions to billions of prolate particles. Therefore, arrays of particles need to be considered in revealing the effect of particle orientations. Li et al. [15] found that $S$ could be used to quantify these orientations. Through systematic DNSs, they have revealed that $S$ has a significant impact on the drag force experienced by arrays of prolate particles. However, the impact of the mean orientation on the heat transfer properties of prolate particles has not been discovered. Therefore, this work aims to quantify this impact and develop new correlation for heat transfer of prolate particles.

In the present work, DNSs of flows past arrays of prolate particles with different $S$ have been performed. The temperature of fluid at the inlet is set different from that of particles to enforce the heat transfer between the two phases. A wide range of solid volume fractions and Reynolds numbers are considered. It has been found that $S$ results in a significant variation in forced convection heat transfer at any specific value of solid volume fraction and Reynolds number. Furthermore, a Nusselt number correlation is proposed in terms of Reynolds number, solid volume fraction, and Hermans orientation factor. It should be mentioned that, as a first step towards exploring the orientation effect on heat transfer from prolate particles, this work focuses on prolate particles with aspect ratio of 2.5 and the proposed correlation is only valid for this specific aspect ratio. The choice of aspect ratio of 2.5 is due to the fact that the only available work in the literature on heat transfer of arrays of prolate particles is performed at this aspect ratio by $\mathrm{He}$ and Tafti only at $S=0$ [14]. Results of the present study have been validated and found to be in good agreement with the results of He and Tafti.

\section{Numerical Method}

2.1. Governing Equations. LBM has been used to simulate various flow phenomena in both two and three dimensions. Generally, the model in LBM is described by $D_{p} Q_{q}$, where index $p$ denotes the number of dimensions in which specific problem is defined and $q$ depicts the number of lattice velocity vectors. In the present work, $D_{3} Q_{19}$ model is used. Mathematical representation of LBM can be expressed in the following form [16]:

$$
\begin{aligned}
f_{i}\left(\mathbf{x}+\mathbf{e}_{\mathbf{i}} \Delta t, t+\Delta t\right)-f_{i}(\mathbf{x}, t)= & -\frac{1}{\tau_{f}}\left(f_{i}(\mathbf{x}, t)-f_{i}^{\mathrm{eq}}(\mathbf{x}, t)\right) \\
& +\Delta t F_{A_{\mathbf{F}, i}}(\mathbf{F}) .
\end{aligned}
$$

In the above equation, $f_{i}$ is the distribution function that shows the histogram representation of frequency of occurrence. These frequencies are the direction-specific fluid densities. $e_{i}$ are the lattice velocities, having the magnitude of 1 or $\sqrt{2} l u / t s$ depending on the values of $i ; f_{i}^{\text {eq }}$ is the 

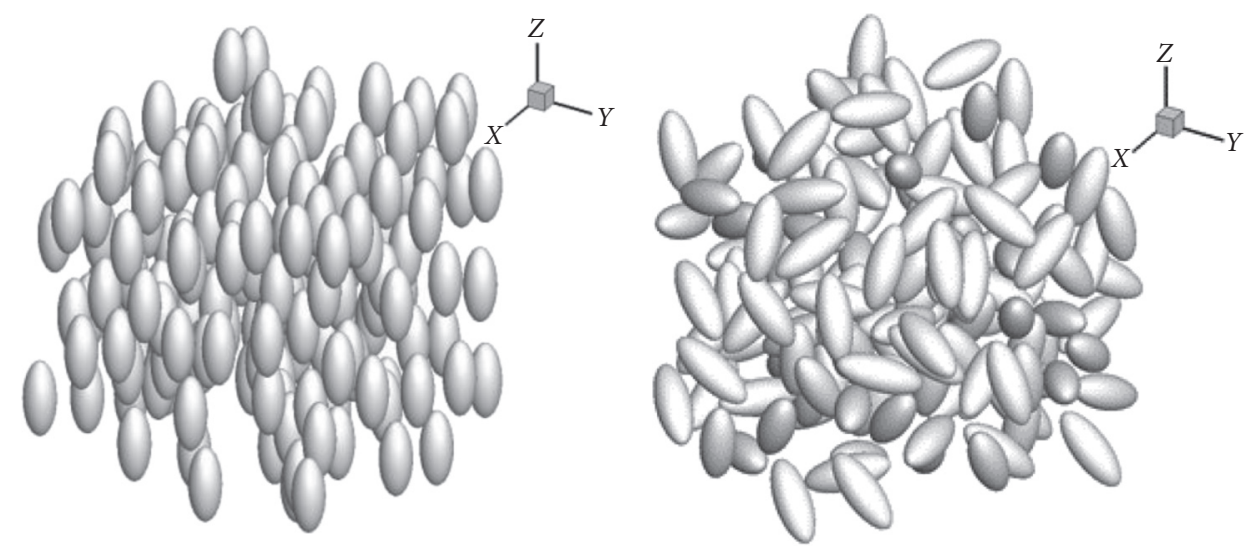

(a)

(b)
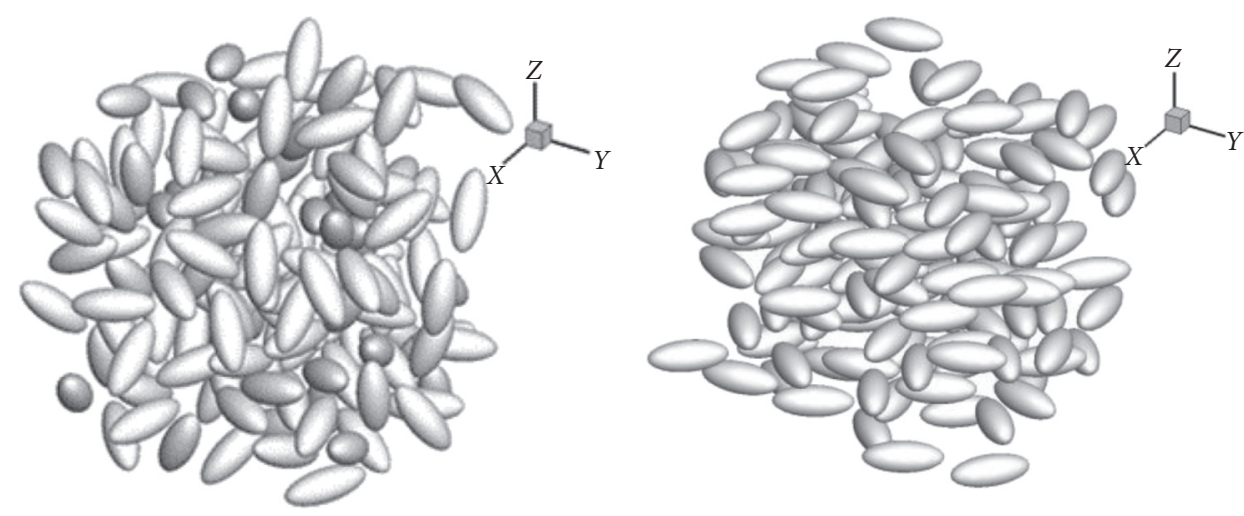

(c)

(d)

FIGURE 1: Arrays of prolate particles with aspect ratio of 2.5, solid volume fraction of 0.2 , and reference direction along $z$ direction: (a) $S=1$, (b) $S=0.2$, (c) $S=0$, and (d) $S=-0.5$.

equilibrium distribution function; $\tau_{f}$ is the relaxation factor. The last term on the right-hand side depicts the effect of force from solid phase to the gas phase. Temperature field in LBM can be obtained by another equation having similar form to that of equation (1) [17]:

$$
\begin{aligned}
g_{i}\left(\mathbf{x}+\mathbf{e}_{\mathbf{i}} \Delta t, t+\Delta t\right)-g_{i}(\mathbf{x}, t)= & -\frac{1}{\tau_{g}}\left(g_{i}(\mathbf{x}, t)-g_{i}^{\mathrm{eq}}(\mathbf{x}, t)\right) \\
& +\Delta t Q_{A_{Q, i}}(Q),
\end{aligned}
$$

where $\tau_{g}$ is the relaxation time and the last term on the righthand side is the heat source term. For details of the numerical methods, reader can refer to [17], since the same computer code is used except that the simulated particles in this work are prolate ellipsoids. The difference between the sphere and prolate model lies in the distribution of Lagrangian markers on the particle surface. In the case of spherical particles, markers are distributed uniformly on the surface of sphere but in the case of prolate particles more markers are present in region with larger surface curvature of the ellipsoids. Different assumptions are made during the simulations and they are given as follows:

(i) Laminar flow (ii) Heat capacity and density of the fluid remain constant throughout the simulations

(iii) Effect of temperature alteration on the velocity and density is not considered

(iv) Effects of radiation and viscous dissipation are not considered

2.2. Nusselt Number Calculations. Nusselt number is defined as the ratio of strength of convection to conductive heat transfer. Its generalized form is [18]

$$
\mathrm{Nu}=\frac{h D}{k},
$$

where $h$ is the convective heat transfer coefficient, $D$ is the particle equivalent diameter, and $k$ is the fluid thermal conductivity. For Reynolds number calculations, equivalent diameter and superficial gas velocities $(U)$ have been used; that is, $\operatorname{Re}=\rho U D / \mu$. In IB-LBM, Nusselt number is calculated in computational domain by making slices of unit thickness in the flow direction. Average temperature of the slice is calculated by [17]

$$
\left\langle T_{f}\right\rangle=\frac{\int_{A} \alpha u_{z}(x, y, z) T(x, y, z) \mathrm{d} x \mathrm{~d} y}{\int_{A} \alpha u_{z}(x, y, z) \mathrm{d} x \mathrm{~d} y} ; \quad \alpha \in[0,1],
$$


where $A$ is the area of slice. Integration is executed over the surface, which is perpendicular to the direction of flow. More details of the heat transfer calculations by this method can be found in the literature [17]. Nusselt number calculations of these slices adopt the following conventions [17]:

$$
\begin{aligned}
h_{f, \text { slice }} & =\frac{Q_{\text {slice }}}{a_{p} V_{\text {slice }}\left(T_{s}-\left\langle T_{f}\right\rangle\right)}, \\
\mathrm{Nu}_{f, \text { slice }} & =\frac{h_{f, \text { slice }} D}{k}, \\
\mathrm{Nu}_{\text {overall }} & =\frac{\sum \mathrm{Nu}_{f, \text { slice }}}{N_{\text {slice }}},
\end{aligned}
$$

where $V_{\text {slice }}, N_{\text {slice }}, a_{p}$, and $Q_{\text {slice }}$ denote volume of slice, number of slices, specific surface area, and heat flux from particles to the fluid phase, respectively.

2.3. Creation of Random Arrays. Random arrays of prolate particles with different Hermans orientation factors are generated by means of the in-house application of Monte Carlo method [19]. The functional form of orientations factors $(S)$ is [15]

$$
S=\frac{\left.3<\cos ^{2} \varnothing\right\rangle-1}{2}, \quad \varnothing \in\left[0, \frac{\pi}{2}\right],
$$

where $\varnothing$ is angle among reference direction and semimajor axis of the prolate particle. Reference direction is defined as the average direction of all particles' semimajor axes in the domain. $S$ takes the values from -0.5 to $1, S=0$ shows random orientations of particles, $S=1$ corresponds to the perfect alignment of prolate particles with respect to reference direction, and $S=-0.5$ represents perfect normal alignment as shown in Figure 1. Through the random generation process of assemblies, particles overlap is prohibited and if such situation occurs, then that location of particle is rejected and a new location is assigned. Solid particle locations are random in nature, so the present method depicts the true picture of natural process in gassolid flows. For different values of Hermans orientation factors, heat transfer studies of prolate particles with aspect ratio ar $=2.5$ under moderate Reynolds number have not thus far been performed in the literature. Aspect ratio and solid volume fractions are defined by [15]

$$
\begin{aligned}
\operatorname{aspect} \text { ratio }(\mathrm{ar}) & =\frac{a}{b},\left\{\begin{array}{ll}
a & \text { prolate semi major axis } \\
\mathrm{b} & \text { prolate semi minor axis }
\end{array},\right. \\
c & =\frac{4 \pi n a b^{2}}{3 L^{3}},\left\{\begin{array}{l}
n \text { number of solid particles } \\
L \text { length of packed cubic section }
\end{array}\right.
\end{aligned}
$$

Prolate particles have three different solid volume fractions, that is, $c=0.1,0.2,0.3$, and for each solid volume fraction four spheroid orientations, that is, $S=\{-0.5,0$, $0.2,1\}$, are studied.

\section{Geometry Description and Code Validation}

3.1. Geometry and Boundary Conditions. Prolate particles with aspect ratio ar $=2.5$ at three solid volume fractions $\{0.1$, $0.2,0.3\}$ are studied. For each solid volume fraction, arrays with four Hermans orientation factors, that is, $S=\{-0.5,0,0.2,1\}$, are simulated. For each solid volume fraction, Reynolds number and Hermans orientation factor results are averaged based on three different configurations. Each configuration represents a realization at the prescribed specific parameters. The simulated Reynolds numbers are confined in the range from 0 to 100 . Choice of this aspect ratio is due to the fact that only existing data in literature are for $S=0$ with ar $=2.5$. In industry applications, for example, in drying process, particles are of smaller size. Depending on particle characteristic length, Reynolds number remains smaller. Therefore, in the present research, range of the Reynolds number has been taken up to 100 . Range of $c$ is selected according to many practical fluidization reactors. The maximum $c$ of 0.3 is chosen because systems with larger $c$ are difficult to generate with a wide range of $S$. Lattice unit system is used in all the simulations. The boundary conditions are the same as those used in [17]. Temperature of the solid particles is specified to be 1 and fluid bulk temperature is fixed at 0 at the inlet. Prandtl number is set equal to unity. It means that hydrodynamic and thermal boundary layers are of the same size. A constant pressure gradient is employed across the domain to drive the flow. At the outlet, temperature gradient was set to zero. Gas flows parallel to increasing $z$ direction. Domain size of $1.4 \mathrm{~L}$ is used, where $\mathrm{L}$ is the side length of packed cubic section. Schematic representation of the complete description of domain is mentioned in Figure 2. Three sizes of grids $d / \Delta x=8,12,16$, are used, where $d$ is the length of the minor axis of the particles. Final results are obtained by Richardson Extrapolation method using three-grid data as shown in Figure 3. This is because many studies, such as $[19,21]$, reported that the use of Richardson Extrapolation method was necessary for the particle-resolved simulations to obtain the grid-independent results. Particle numbers used for different solid volume fractions are in the range of 120 to 189. More than 500 numerical simulations were performed during this study.

3.2. Code Validation. Huang et al. have already validated the present code of IB-LBM for spherical single as well as arrays of spherical particles for variety of solid volume fractions [17]. Before doing the heat transfer calculations for arrays of ellipsoidal particles, code validation of single ellipsoidal particle is performed. Richter and Nikrityuk performed the heat transfer calculations of cubes and ellipsoids in flows using ANSYS FLUENT software package. They simulated two cases for ellipsoids: one is parallel to the flow direction; that is, major axis of the ellipsoids was set parallel to the flow (ellipsoid 1) or perpendicular to the flow (ellipsoid 2). Therefore, to check the validity of present code, singleprolate particle is simulated for two orientations: one is parallel and other is perpendicular to the flow direction. 


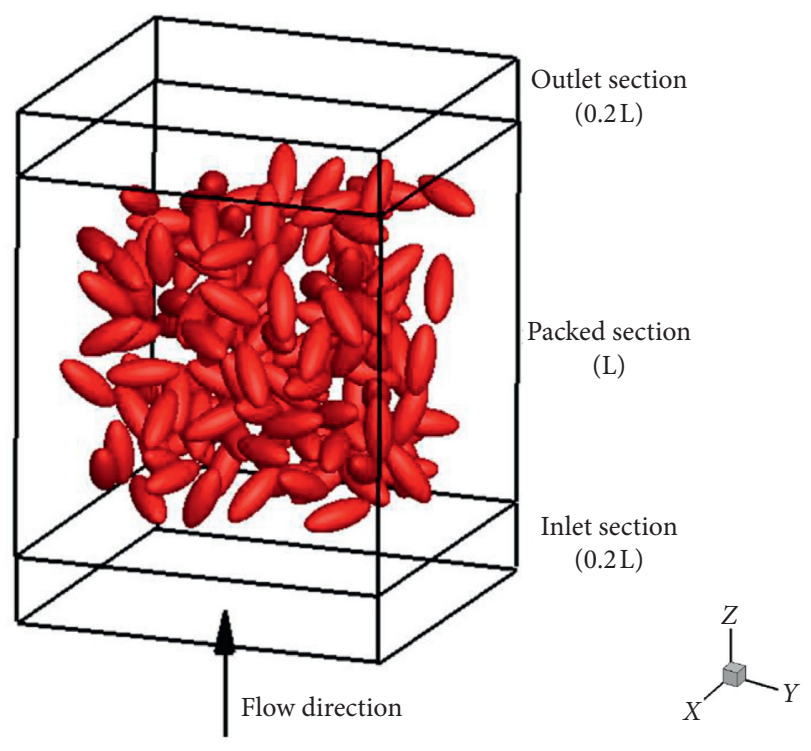

FIGURE 2: Schematic representation of computational domain $(\operatorname{ar}=2.5, S=0$, and $c=0.2$ ).

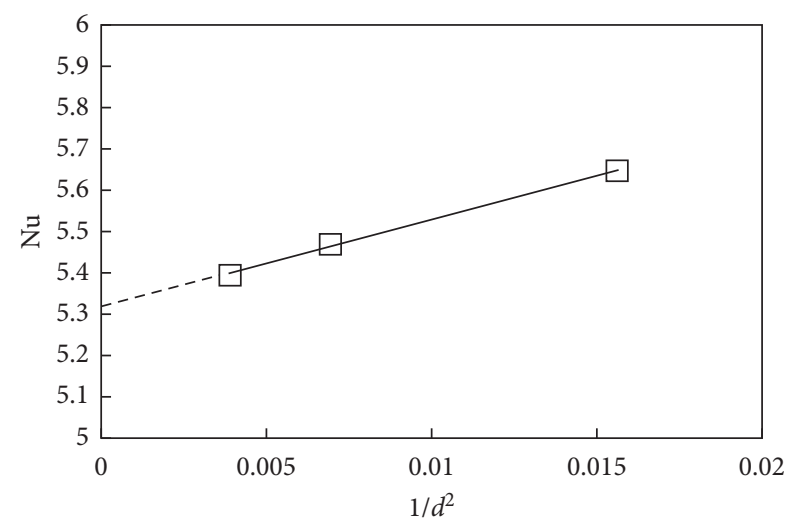

FIGURE 3: Three symbols are the three grids' values and the intersection between the dashed line and the vertical axis is the Richardson Extrapolation result depending on the three known grid values. The shown results are from a configuration of prolate particles at $\operatorname{Re}=24, c=0.1$, and $S=0.2$.

Here, IB-LBM results are compared with the reported data of Richter and Nikrityuk. Nusselt number of single ellipsoidal particle is obtained by extrapolation at three small solid volume fractions of $0.01,0.02$, and 0.03 . Simulation results are presented in Figure 4 with the results of Richter and Nikrityuk [10]. The results are found to be in good agreement with maximum deviation of less than $3 \%$ from the literature results. The comparison depicts that IB-LBM can produce the results in good accuracy when compared with commercial computational fluid dynamic software package.

For further validation, arrays of prolate particles at ar $=2.5$ and $S=0$ are considered. Figure 5 shows variation of Nusselt number versus Reynolds number for different solid volume fractions. Results show that Nusselt number increases with the increase of Reynolds number. The results of

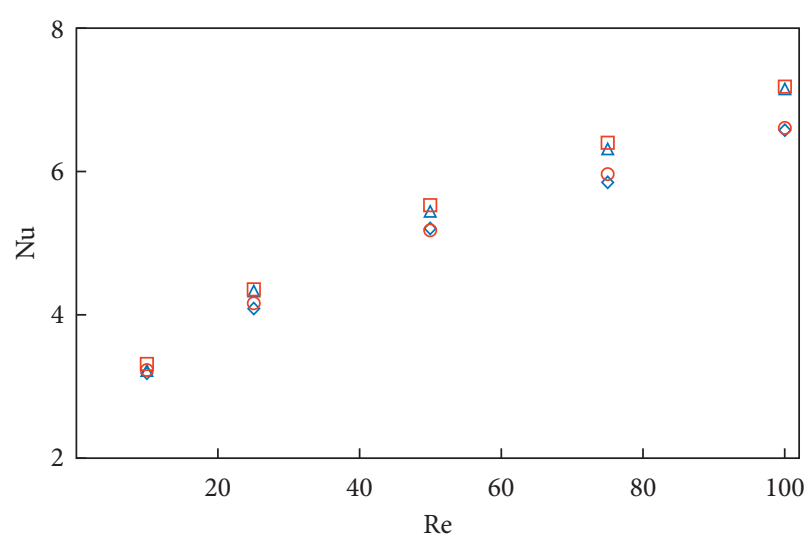

$\diamond$ Ellipsoid 1 present result
$\Delta$ Ellipsoid 2 present result
$\circ$ Ellipsoid 1 Richter and Nikrityuk (2012)
$\square$ Ellipsoid 2 Richter and Nikrityuk (2012)

FIgURE 4: Single ellipsoidal particle. Ellipsoid 1 parallel to flow direction and ellipsoid 2 perpendicular to flow direction.

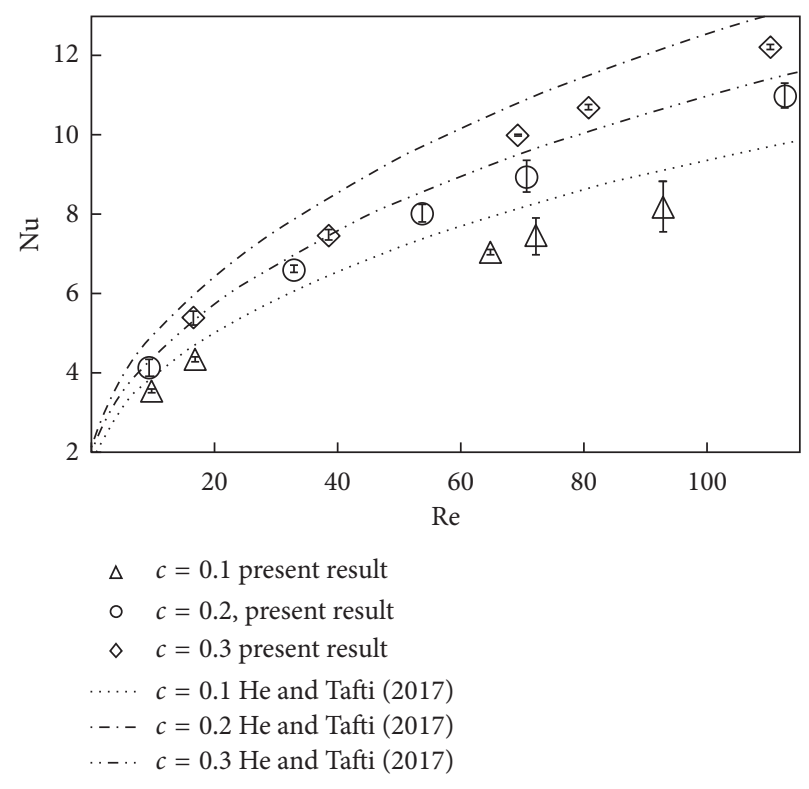

FIGURE 5: Nusselt number variation with respect to Reynolds number for various solid volume fractions at $\operatorname{ar}=2.5$ and $S=0$. Error bars represent standard deviation in Nusselt number obtained from three different samples of random configurations.

the present study are compared with those of He and Tafti [14] who also simulated the prolate particles for solid volume fractions of 0.1 to 0.35 . In general, the results are in good agreement with the literature both qualitatively and quantitatively. Minor differences are due to the use of different type of immersed boundary method in the present study. Also, $\mathrm{He}$ and Tafti have used constant heat flux boundary condition, whereas constant temperature boundary condition has been employed in the present research work. It is a known fact that the Nusselt number is lower for constant 
temperature boundary case as compared to constant heat flux boundary conditions due to lower effective temperature gradient at the wall [18].

\section{Results and Discussion}

Average Nusselt number at the start of simulations has higher value but after some time steps it achieves its steady state as shown in Figure 6. During the process of forced convection heat transfer from hot particles, the temperature of gas increases as the gas approaches the outlet section as shown in Figure 7. The temperature attained near the outlet of the domains depends upon the Re, solid volume fraction, and $S$. As the temperature of fluid rises along the fluid direction, the potential for heat transfer decreases as temperature gradient at the particle surfaces is decreased.

Reynolds number plays a significant role in heat transfer phenomenon because it is the driving force behind the forced convection. As flow velocity (or Re) is increased, the fluid temperature in the cross section decreases, as shown in Figure 8, due to enhanced heat transfer.

As the ellipsoidal objects are $3 \mathrm{D}$ in nature, the orientations of these particles may modify the flow field around the assembly of particles. Therefore, orientations of these particles in the assembly can significantly influence the heat transfer. To investigate these effects, numerical simulations are performed for various Hermans orientation factors. For parallel arrays with $S=1$, all particles are positioned such that they are parallel to the flow direction. Figure 9 ellipsoidal particles wh depicts that Nusselt number increases with the increase of Reynolds number due to enhanced convective heat transfer at higher Re. At a fixed Re, Nusselt number increases with solid volume fraction because the small interstitial spaces among the particles cause flow velocity to increase, which enhances the forced convection heat transfer as mentioned by [22].

The results for $S=0.2$ are shown in Figure 10. Variations of $\mathrm{Nu}$ in the cases of $S=0.2$ and $S=1$ are not the same because of the different local recirculation zones due to particles orientations. Figure 11 shows the Nusselt number variation versus Reynolds number for $S=-0.5$. For this orientation factor, flow direction and major axes of ellipsoids are normal to each other. Here, difference between Nusselt numbers of two consecutive solid volume fractions is smaller because there is a negligible variation in flow velocity as the solid volume fraction is altered. However, at any fixed Reynolds number, solid volume fractions cause $\mathrm{Nu}$ number to increase.

4.1. Influence of Hermans Orientation Factor on Nusselt Number. Nusselt number is the dimensionless temperature gradient at the surface of particle. Usually heat transfer correlations of Nusselt number are developed using experimental results. However, for arrays of particles, it is difficult and economically expensive to calculate the heat transfer coefficient experimentally. In functional form, it has dependency on the Reynolds number and Prandtl number for single-particle case. But, for multiparticle system, effect of solid volume fraction and its orientation cannot be

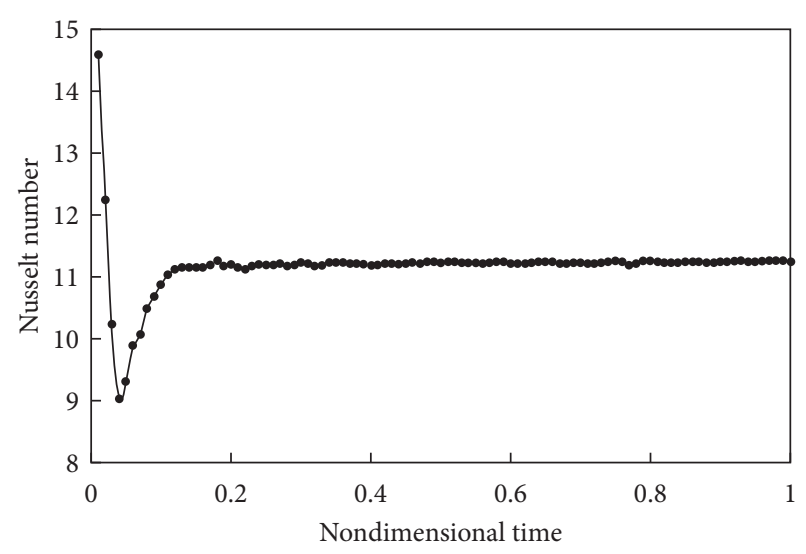

Figure 6: Average Nusselt number with Hermans orientation factor of 0.2 , with $c=0.3$ and $\operatorname{Re}=82$.

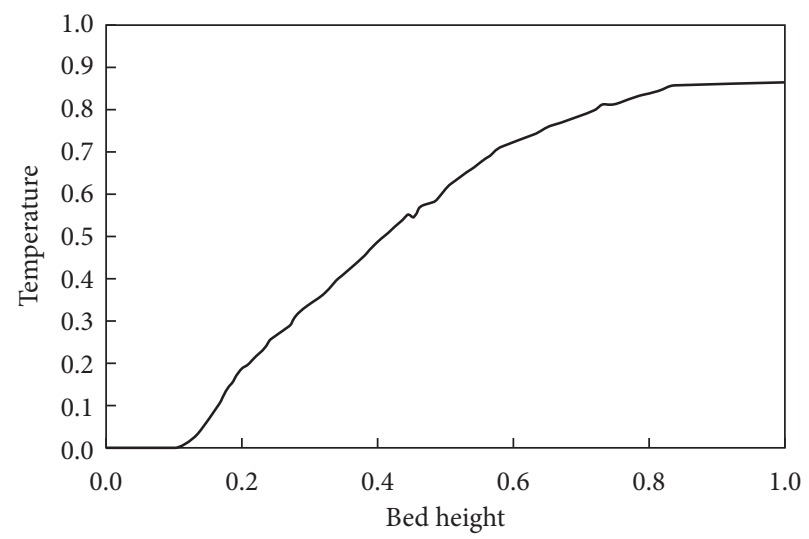

FIgURE 7: Gas temperature rise with bed height. $S=-0.5, c=0.1$, and $\operatorname{Re}=12.72$.

neglected. Therefore, $\mathrm{Nu}=f(\operatorname{Re}, \operatorname{Pr}, c, S)$. By using the simulation data, a correlation has been developed for assembly of ellipsoidal particles with aspect ratio of 2.5 and it reads

$$
\begin{aligned}
\mathrm{Nu}= & \left(1.5-0.885(1-c)+0.078(1-c)^{2}\right) \\
& \cdot\left[2.458-0.042 \operatorname{Re}^{1.07} \operatorname{Pr}^{1 / 3}\right] \\
& +\left(1.115-0.62(1-c)-0.08(1-c)^{2}\right) \operatorname{Re}^{0.68} \operatorname{Pr}^{1 / 3} \\
& +(-S+0.08) .
\end{aligned}
$$

Equation (8) is valid for $0<\operatorname{Re} \leq 100,0.1 \leq c \leq 0.3$, and $S=\{-0.5,0,0.2,1\}$ for solid particles with aspect ratio of 2.5. The coefficients of equation (8) are determined by minimizing the difference between the simulated data and predicted correlation. Predicted values of Nusselt number using equation (8) are plotted with simulation results in Figure 12. It can be seen that the proposed correlation gives satisfactory performance and the maximum deviation from the simulated results is less than $10 \%$. 


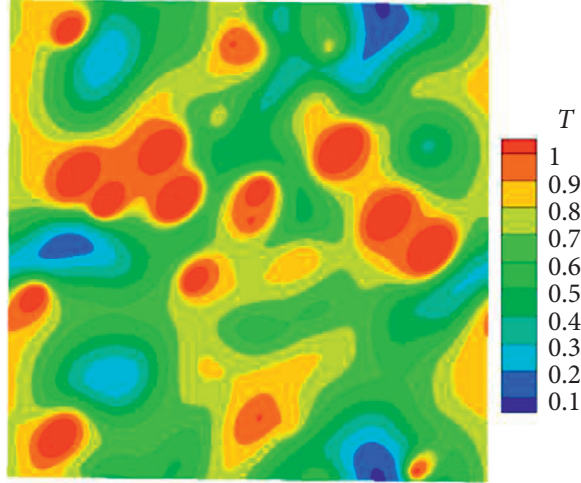

(a)

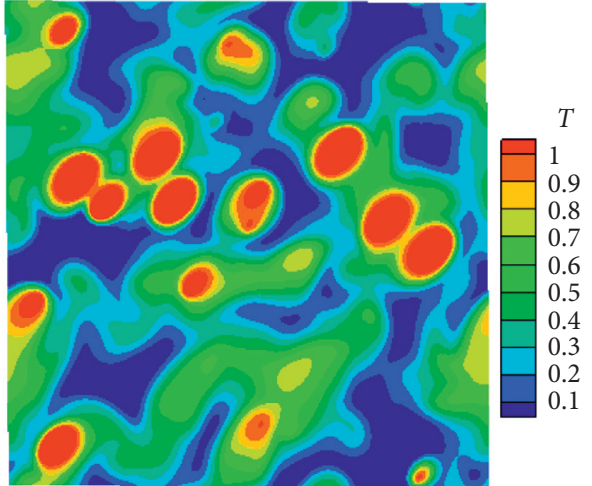

(b)

Figure 8: Temperature contours in $x y$ plane (middle of packed section), $S=0$ at 0.1 solid volume fractions. (a) $\operatorname{Re}=16.5$ and (b) $\operatorname{Re}=90$.

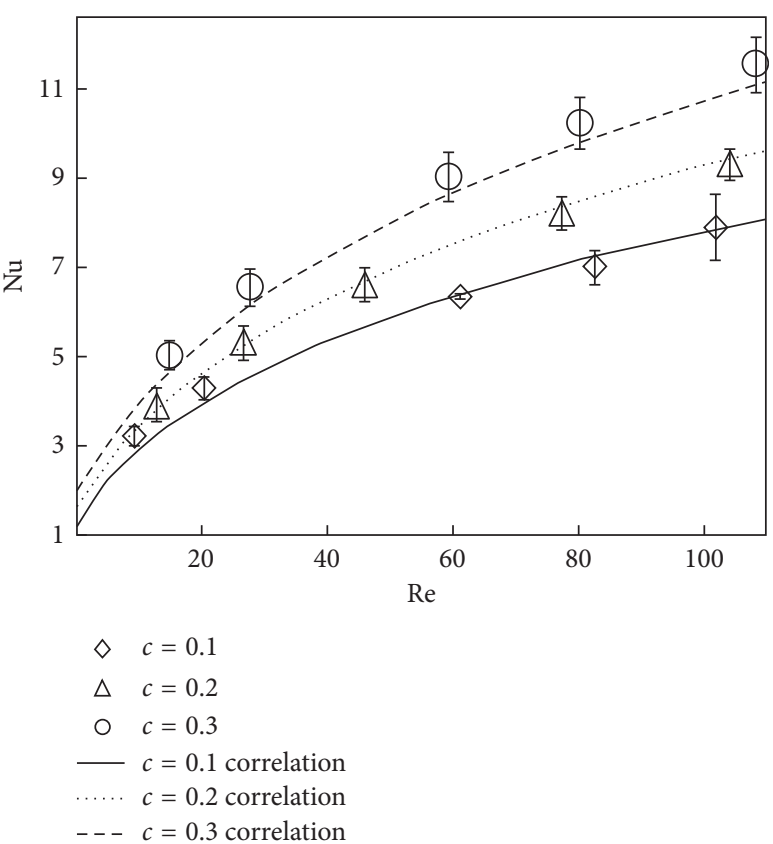

FIGURE 9: Nusselt number variation with Reynolds numbers for ar $=2.5$ and $S=1$ at $c=0.1,0.2$, and 0.3 . Symbols represent simulation results and lines obtained from correlation in equation (8).

Impact of Hermans orientation factor on Nusselt number can be probed by using equation (8).

Effects of orientations cannot be neglected in forced convection heat transfer because this phenomenon strongly depends on the flow field variations. For different values of $S$, Nusselt number varying with the Reynolds number is plotted in Figure 13. Only the results at $c=0.2$ are shown as illustrative examples. It can be seen that the Nusselt number generally decreases with the increase of $S$. The proposed correlation agrees favorably well with the DNS results. Also the variation of Nusselt number with Hermans orientation factors is presented in Figures 14(a) to 14(d) at various Reynolds numbers. Figure 14(a) is the plot at Reynolds number of 20, which shows that $\mathrm{Nu}$ decreases with the increase of $S$ linearly at various solid volume fractions. This

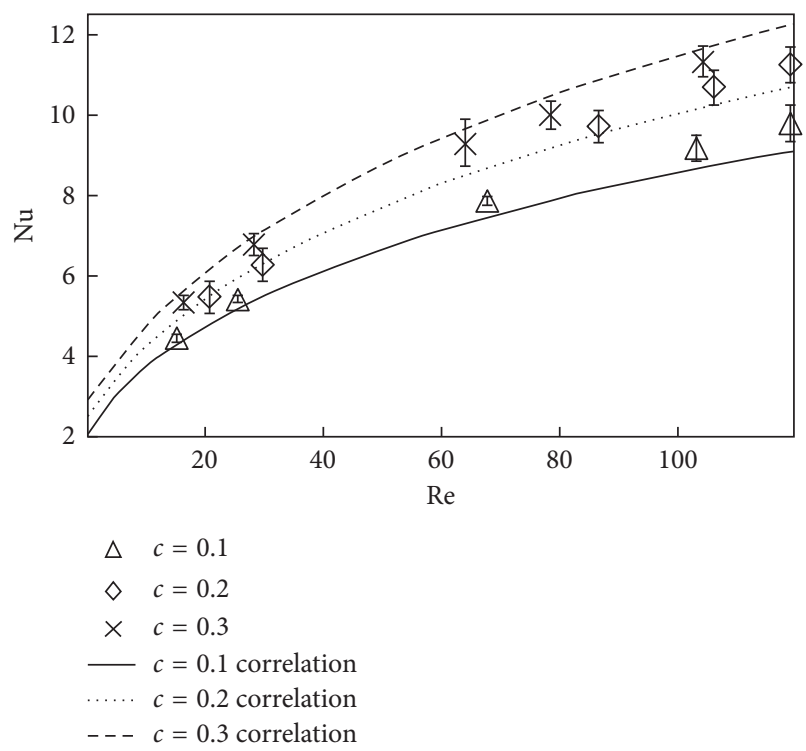

FIgURE 10: Nusselt number versus Reynolds number for ar $=2.5$ and $S=0.2$ at $c=0.1,0.2$, and 0.3 . Symbols represent simulation results and lines obtained from correlation in equation (8).

trend is also observed at other Reynolds numbers, since the proposed equation (8) is indeed a linear function of $S$ when other parameters in the correlation are fixed.

Nusselt numbers at $S=1$ have minimum values as compared to the other three orientations at the same solid volume fractions. This is due to the less recirculation of flow as particles are aligned along the flow direction.

Figure 14 shows that the effect of $S$ on average Nusselt number is very significant and this behavior remains obvious even with the increase of Reynolds number. It can be realized from Figure 14(a) that, at the Reynolds number of 20, the value of Nusselt number at $c=0.1$ and $S=-0.5$ is almost $9 \%$ more than the respective value at $S=0$. This may be due to the fact that, for lower solid volume fraction $(c=0.1)$, $S=-0.5$ gives rise to higher value of the effective heat transfer surface area as compared to $S=0$. Moreover, when compared to $S=0.2$, Nusselt number for $S=-0.5$ has $13 \%$ higher 


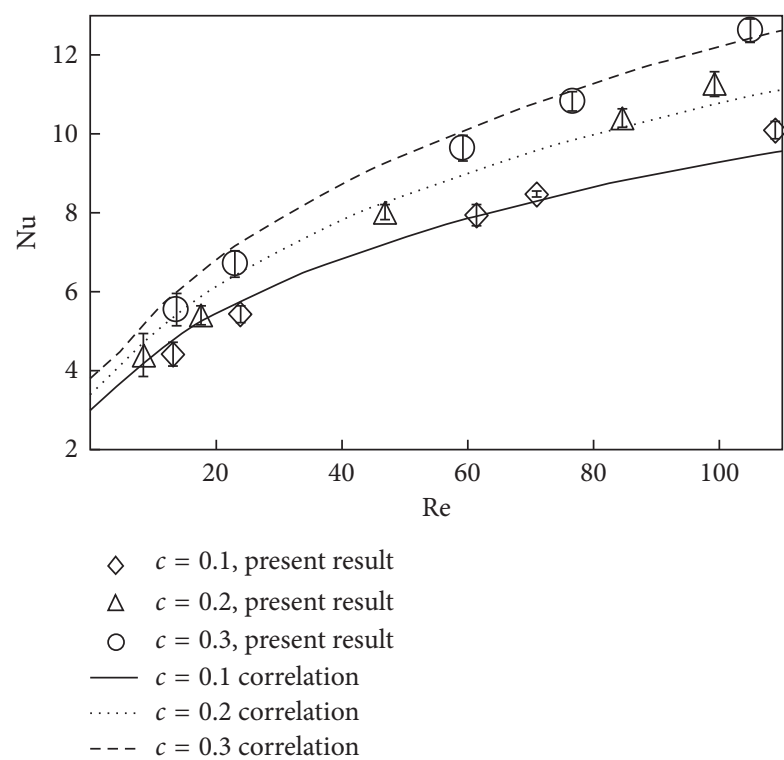

Figure 11: Nusselt number versus Reynolds number for ar $=2.5$ and $S=-0.5$ at $c=0.1,0.2$, and 0.3 . Symbols represent simulation results and lines obtained from correlation in equation (8).

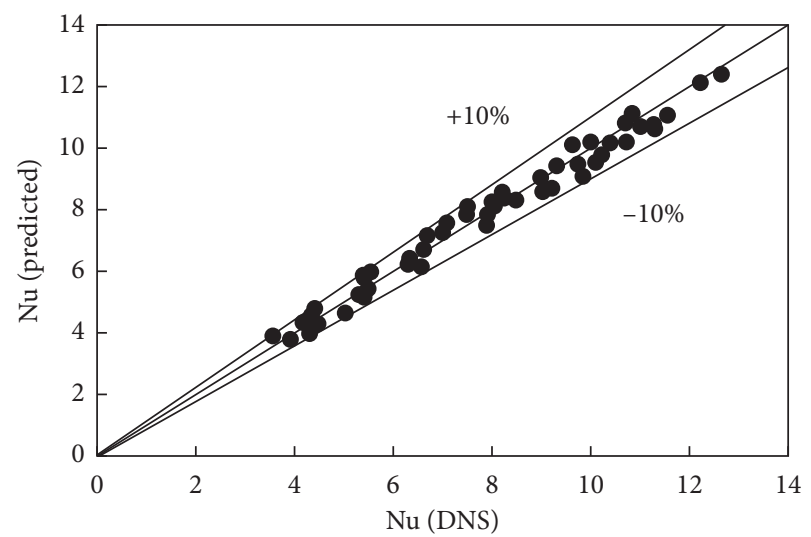

FIGURE 12: Comparison of average Nusselt number from DNS with correlation predicted values.

value. Similarly, the forced convection strength of $S=-0.5$ is $27 \%$ higher when compared with $S=1$ at $c=0.1$. Highest value of Nusselt number occurs for $c=0.1$ and $S=-0.5$ is due to the impact of jet and enhanced recirculation. Also, the ellipsoidal particles whose major axis is placed parallel to the flow direction have smaller $\mathrm{Nu}$ as mentioned in [23].

For $c=0.2$ and $\operatorname{Re}=50$ in Figure 14(b), it can be observed that prolate particle assembly with $S=-0.5$ again has the highest heat transfer properties as compared to the other orientations. This increase is $6 \%, 8 \%$, and $17 \%$ as compared to $S=0, S=0.2$, and $S=1$, respectively. It is observed that, with increase of $c$, maxima was again achieved at $S=-0.5$. The amount of heat transfer from each assembly depends on many factors including heat transfer surface area, local flow velocity, temperature gradient, and vortices formed near the solid particle [24].

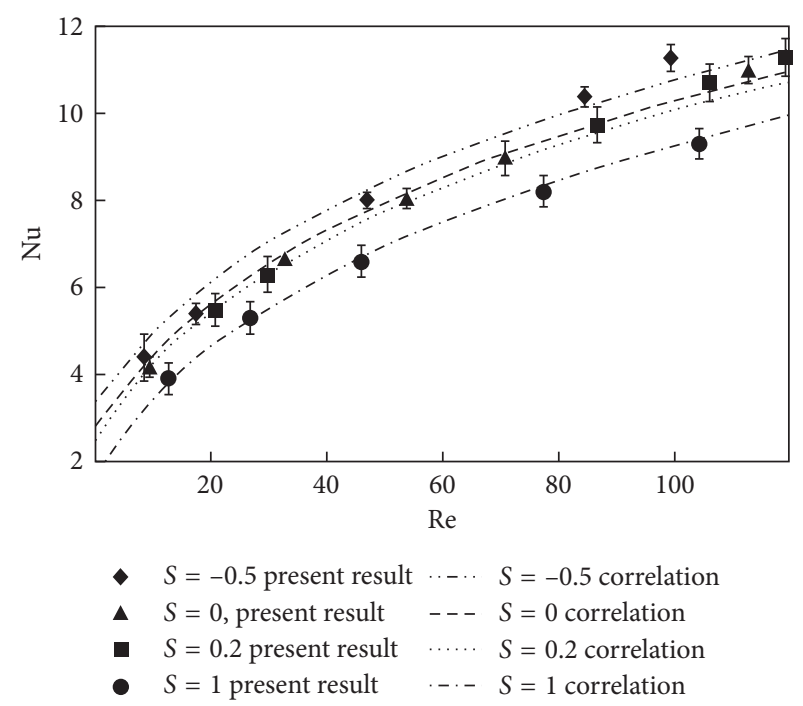

FIGURE 13: Nusselt number variation for different Hermans orientation factor $c=0.2$.

Figures 14(c) and 14(d) show effect of $S$ on Nusselt number at Reynolds numbers of 90 and 100, respectively. For $c=0.3$ and $S=-0.5$, average Nusselt number is $4 \%$ more than the random orientation $(S=0)$ case. Therefore, it can be concluded that arrays of particles whose major axes are perpendicular to the flow direction have high value of Nusselt number $\left(\mathrm{Nu}_{S=-0.5)}\right.$ as compared to the other orientations. Parallel arrays have minimum values of Nusselt number $\left(\mathrm{Nu}_{S=1}\right)$. Overall percent increase in Nusselt number caused by $S$ can be calculated by using equation (9) and is plotted in Figure 15 for different values of solid volume fractions and Reynolds number.

$$
\% \text { increase }=\frac{\mathrm{Nu}_{S=-0.5}-\mathrm{Nu}_{S=1}}{\mathrm{Nu}_{S=-0.5}} \times 100 .
$$

Figure 16 represents the contours at 0.2 solid volume fractions for three different values of $S$. All the three cases have distinct local structure that results in unique convective heat transfer behavior. For $S=1$, the temperature of the gas around the particles is still lower due to less heat transfer as compared to the other two cases.

4.2. Stanton Number. Stanton number, St, also known as Margoulis number $(M)$, is another dimensionless number that is used in forced convection heat transfer process. It measures the ratio of heat transported into a fluid to the thermal capacity of the fluid. Mathematical expression for this number is [18]

$$
\mathrm{St}=\frac{\mathrm{Nu}}{\mathrm{RePr}}
$$

In the present study, Prandtl number is 1 and Stanton number can also be calculated from the simulation data. Dimensionless numbers have great significance in fluid mechanics. They help in easy parametric investigation of diverse engineering problems. Figure 17 shows the trend of 

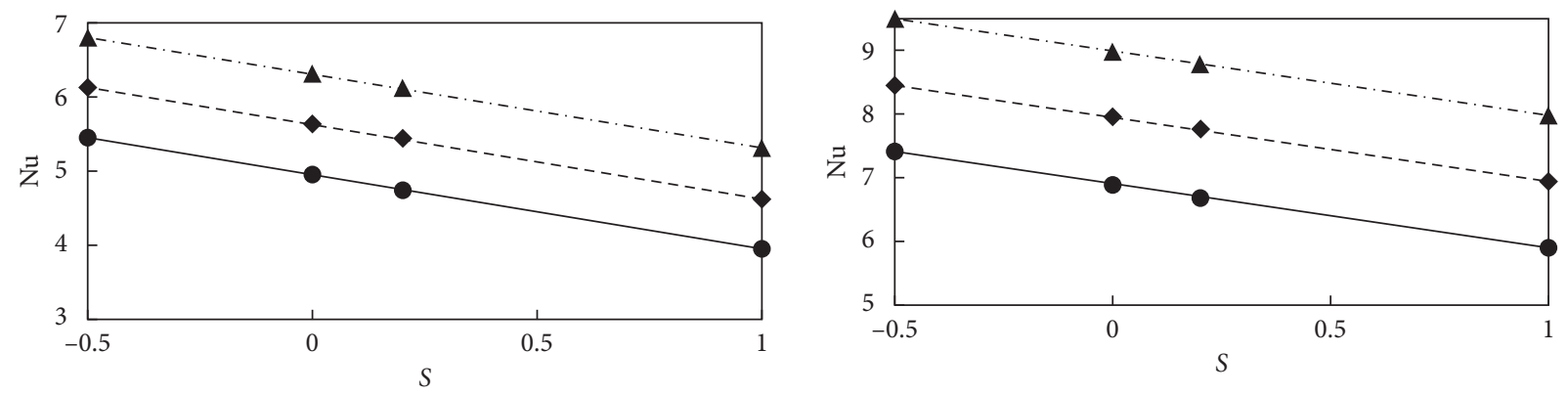

$c=0.1$
$c=0.2$
$c=0.3$
- $c=0.1$
$c=0.2$
$c=0.3$
$\Delta c=0.3$

(a)

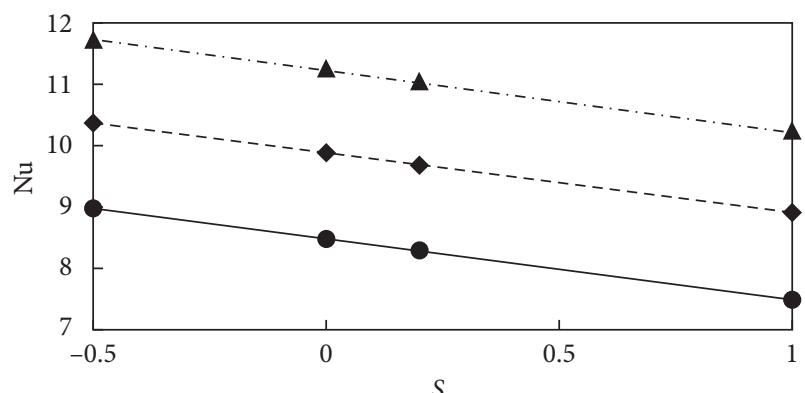

$S$

$\begin{aligned} c & =0.1 \\ c & =0.2 \\ -c & =0.3\end{aligned}$

(c)

(d)

Figure 14: Nusselt number variation with Hermans orientation factor. (a) $\operatorname{Re}=20$, (b) $\operatorname{Re}=50,(c) \operatorname{Re}=90$, and $(d) \operatorname{Re}=100$.

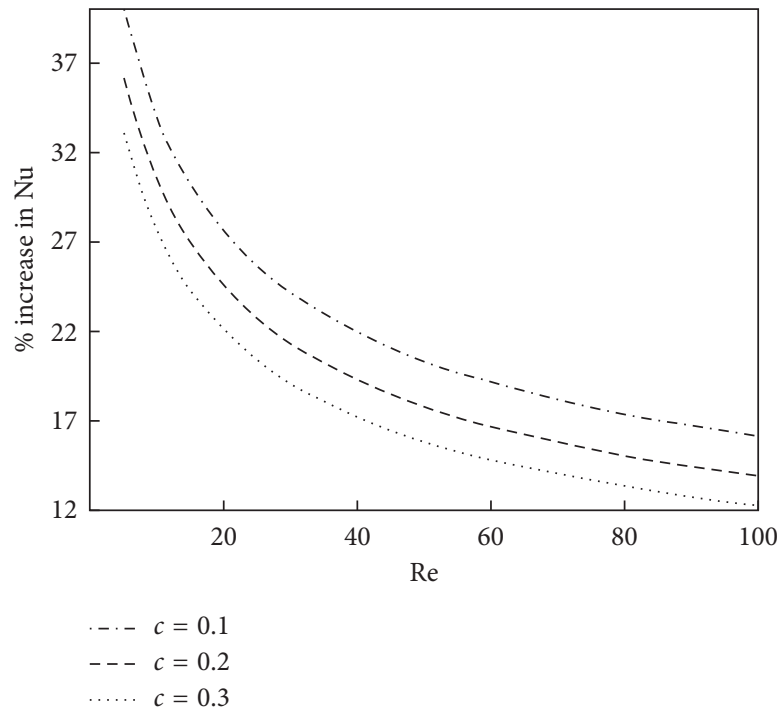

Figure 15: Percent increase in Nusselt number caused by $S$ for various solid volume fractions.

Stanton number with Reynolds number. Also Figure 18 shows the effects of Hermans orientation factors on Stanton number at $c=0.2$. Clearly, Stanton number decreases with the increase of $S$ at fixed value of $c=0.2$. Close observation shows that the impact of $S$ on Stanton number decreases with the increase of Reynolds number. The trend observed at other solid volume fractions is similar to that reported at $c=0.2$ and hence is omitted. 


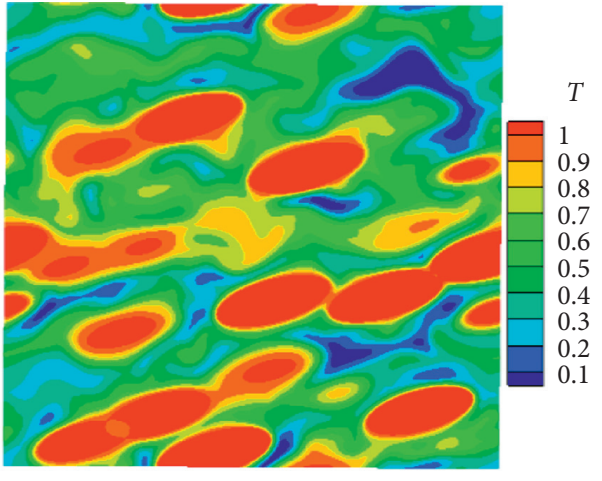

(a)

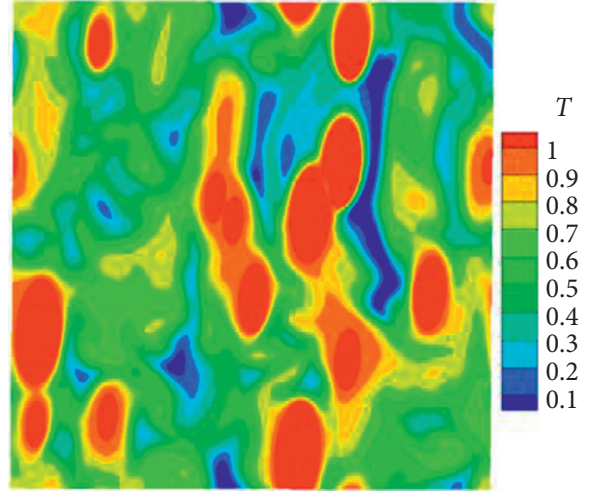

(b)

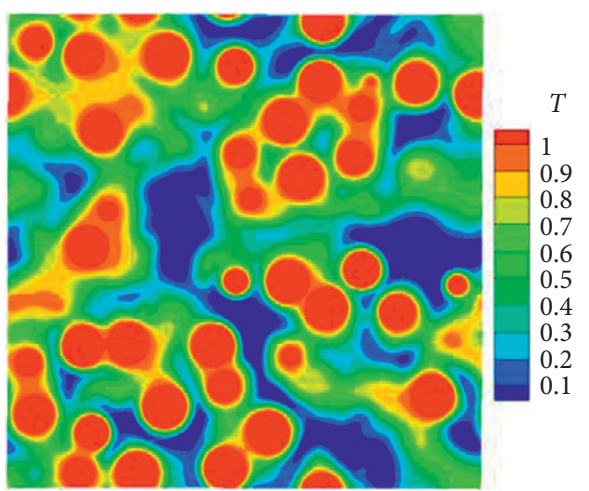

(c)

Figure 16: Temperature contours in $x y$ plane (middle of packed section), $c=0.2$. (a) $\operatorname{Re}=98.9$ and $S=-0.5$. (b) $\operatorname{Re}=90.5$ and $S=0$. (c) $\operatorname{Re}=100$ and $S=1$.
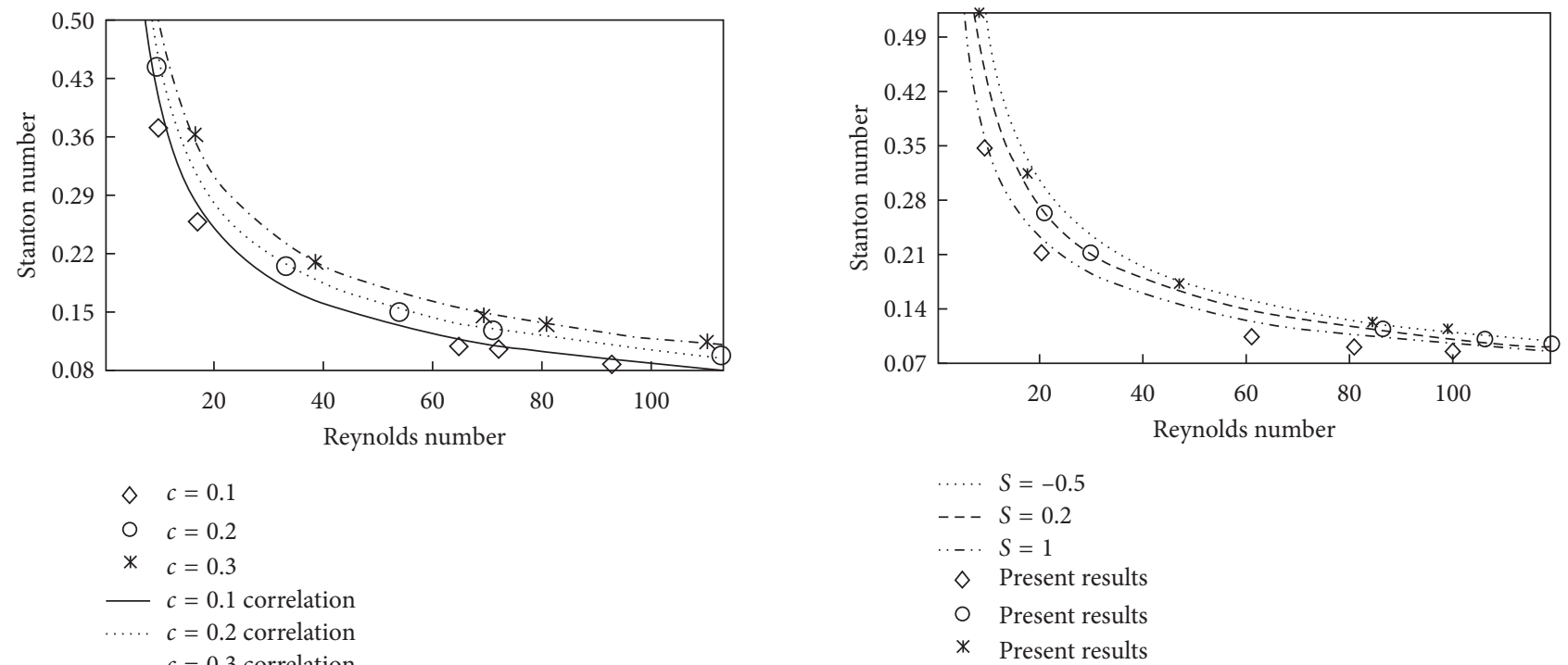

FIGURE 17: Variation of Stanton number with Reynolds number at $S=0$.

Stanton number is also widely used in Reynolds analogy concept of boundary layer theory. From the information of Stanton number, the friction coefficient can be calculated because $\mathrm{St}=C_{f} / 2$ [18]. This analogy is applicable only if

FIGURE 18: Variation of Stanton number for different $S$ at $c=0.2$.

Prandtl number is one and pressure gradient is zero in limiting condition. However, this analogy can be applied with its limitations just to have the first approximation of the magnitudes of flow variables, that is, friction or mass flow rate coefficients. So, heat transfer data can be utilized to have 
a good insight into the underlying physical problems. Although this analogy will not give the accurate results, it can provide a firsthand engineering analysis [25].

\section{Summary and Conclusions}

Heat transfer study of prolate particle arrays is performed using second-order accurate Immersed Boundary-Lattice Boltzmann Method (IB-LBM) [16]. Particles with aspect ratio of 2.5 are studied. Arrays are generated for solid volume fraction from 0.1 to 0.3 by using Monte Carlo method [15]. Moreover, four different values of Hermans orientation factor in the range of -0.5 to 1 are considered. Results are presented by doing averaging on three different random arrays of prolate particles. It is concluded that Nusselt number considerably increases with the increase of Reynolds number and solid volume fractions. Also, Nusselt number significantly decreases with the increase of Hermans orientation factor. For example, at $c=0.1$ and $\operatorname{Re}=20$, Nusselt number of arrays with $S=-0.5$ is approximately $27 \%$ higher than that in arrays with $S=1$. The difference between Nusselt number obtained in arrays of $S=-0.5$ and that obtained in arrays of $S=1$ decreases as the solid volume fraction and Reynolds number increase. This difference is still remarkable at $c=0.3$ and $\mathrm{Re}=100$, which is around $12 \%$.

Based on the present simulations, a correlation is presented for heat transfer of prolate particles. The maximum deviation of the proposed correlation from the simulation results is less than 10 percent. It is noted that Nusselt number is a linear function of $S$ when other parameters such as $\operatorname{Re}, \operatorname{Pr}$, and $c$ in the correlation are fixed. The proposed correlation would prove beneficial for future studies in practical processes. Stanton number calculations are also performed based on the proposed correlation for Nusselt number. It is found that the impact of $S$ on Stanton number is nonnegligible. Stanton number decreases with the increase of $S$. The impact of $S$ on Stanton number decreases with the increase of Reynolds number. Overall, the present study shows that the effect of mean particle orientation on $\mathrm{Nu}$ for arrays of prolate particles is significant. This indicates that, in practical systems with nonspherical particles, the consideration of the effect of orientation is necessary for accurate prediction of the heat transfer property of the flow. Forthcoming studies will comprise the heat transfer of prolate arrays for several aspect ratios. Consideration of a variety of aspect ratios will be fruitful in biomass applications because in such process enormous particles' aspect ratios exist. In practical applications, particles form different orientations; therefore, the impact of orientations on forced convection for a range of aspect ratio will contribute a lot on the road to the engineering community. In the future, a unified Nusselt number correlation will be presented in the form of aspect ratios, Hermans orientation factor, Reynolds number, and solid volume fraction. The impacts of the orientation on gas-solid heat transfer at even higher Reynolds number and various aspect ratios are recommended for future studies.

\section{Nomenclature}

c: $\quad$ Solid volume fraction

D: $\quad$ Particle equivalent diameter

U: $\quad$ Superficial gas velocities

Re: $\quad$ Reynolds number

$\rho: \quad$ Density of gas

$\mu$ : $\quad$ Viscosity of gas

$S: \quad$ Hermans orientation factors

$f_{i}, g_{i}: \quad$ Distribution functions

$f_{i}^{\text {eq }}, g_{i}^{\text {eq }}$ : Equilibrium distribution functions

$e_{i}: \quad$ Lattice velocity

$x$ : $\quad$ Position vector

$\tau_{f}, \tau_{g}: \quad$ Relaxation factors

$F_{A_{F, i}}(F)$ : Source term in momentum equation

$Q_{A_{Q, i}}(Q)$ : Source term in heat equation

$k: \quad$ Fluid thermal conductivity

$h$ : Convective heat transfer coefficient

Pr: $\quad$ Prandtl number

Nu: $\quad$ Nusselt number

$\mathrm{Nu}_{f \text {,lice: }}$ Nusselt number of slice

$T_{s}: \quad$ Ellipsoid temperature

$\left\langle T_{f}\right\rangle$ : $\quad$ Average temperature of slice

$V_{\text {slice }}$ : Volume of slice

$N_{\text {slice }}$ : Number of slices

$a_{p}: \quad$ Specific surface area

$Q_{\text {slice }}$ : Heat flux from particles to the fluid phase

$h_{f \text {,slice: }}$ Slice heat transfer coefficient

A: $\quad$ Area of slice

$\alpha: \quad$ Signed level set function

$u_{z}: \quad$ Gas velocity in flow direction

$\varnothing$ : $\quad$ Angle

$a, b$ : Particle semimajor and minor axis

ar: $\quad$ Aspect ratio

$n$ : $\quad$ Number of solid particles

L: $\quad$ Length of cubic computational domain

St: Stanton number

$C_{f}$ : Skin friction.

\section{Data Availability}

The data used to support the findings of this study are available from the corresponding author upon request.

\section{Conflicts of Interest}

The authors declare that they have no known conflicts of interest or personal relationships that could have appeared to influence the work reported in this paper.

\section{Acknowledgments}

This computation was supported by HPC Platform, Xi'an Jiaotong University. The authors acknowledge the financial support by the National Natural Science Foundation of China (21978228 and 22078255), Shaanxi Creative Talents Promotion Plan-Technological Innovation Team (2019TD039), the Fundamental Research Funds for the Central Universities (Creative Team Plan no. cxtd2017004 in Xi'an 
Jiaotong University), and the China Scholarship Council (CSC, 2017GXZ021009).

\section{References}

[1] J. Brix, P. A. Jensen, and A. D. Jensen, "Modeling char conversion under suspension fired conditions in $\mathrm{O}_{2} / \mathrm{N}_{2}$ and $\mathrm{O}_{2} / \mathrm{CO}_{2}$ atmospheres," Fuel, vol. 90, no. 6, pp. 2224-2239, 2011.

[2] A. Panahi, Y. A. Levendis, N. Vorobiev, and M. Schiemann, "Direct observations on the combustion characteristics of Miscanthus and Beechwood biomass including fusion and spherodization," Fuel Processing Technology, vol. 166, pp. 41-49, 2017.

[3] D. J. Gunn, "Transfer of heat or mass to particles in fixed and fluidised beds," International Journal of Heat and Mass Transfer, vol. 21, no. 4, pp. 467-476, 1978.

[4] N. Wakao, S. Kaguei, and T. Funazkri, "Effect of fluid dispersion coefficients on particle-to-fluid heat transfer coefficients in packed beds," Chemical Engineering Science, vol. 34, no. 3, pp. 325-336, 1979.

[5] N. G. Deen, S. H. L. Kriebitzsch, M. A. van der Hoef, and J. A. M. Kuipers, "Direct numerical simulation of flow and heat transfer in dense fluid-particle systems," Chemical Engineering Science, vol. 81, pp. 329-344, 2012.

[6] H. Tavassoli, E. A. J. F. Peters, and J. A. M. Kuipers, "Direct numerical simulation of non-isothermal flow through dense bidisperse random arrays of spheres," Powder Technology, vol. 314, pp. 291-298, 2017.

[7] S. Das, N. G. Deen, and J. A. M. Kuipers, “A DNS study of flow and heat transfer through slender fixed-bed reactors randomly packed with spherical particles," Chemical Engineering Science, vol. 160, pp. 1-19, 2017.

[8] K. Suzuki, T. Kawasaki, N. Furumachi, Y. Tai, and M. Yoshino, "A thermal immersed boundary-lattice Boltzmann method for moving-boundary flows with Dirichlet and Neumann conditions," International Journal of Heat and Mass Transfer, vol. 121, pp. 1099-1117, 2018.

[9] G. Juncu, "Unsteady heat transfer from an oblate/prolate spheroid," International Journal of Heat and Mass Transfer, vol. 53, no. 17-18, pp. 3483-3494, 2010.

[10] A. Richter and P. A. Nikrityuk, "Drag forces and heat transfer coefficients for spherical, cuboidal and ellipsoidal particles in cross flow at sub-critical Reynolds numbers," International Journal of Heat and Mass Transfer, vol. 55, no. 4, pp. 13431354, 2012.

[11] H. Zhang, L. Zhang, X. An, and A. Yu, "PR-DNS on the momentum and heat transfer of a rotating ellipsoidal particle in a fluid," Powder Technology, vol. 373, pp. 152-163, 2020.

[12] J. Yang, Q. Wang, M. Zeng, and A. Nakayama, "Computational study of forced convective heat transfer in structured packed beds with spherical or ellipsoidal particles," Chemical Engineering Science, vol. 65, no. 2, pp. 726-738, 2010.

[13] H. Tavassoli, E. A. J. F. Peters, and J. A. M. Kuipers, "Direct numerical simulation of fluid-particle heat transfer in fixed random arrays of non-spherical particles," Chemical Engineering Science, vol. 129, pp. 42-48, 2015.

[14] L. He and D. K. Tafti, "Heat transfer in an assembly of ellipsoidal particles at low to moderate Reynolds numbers," International Journal of Heat and Mass Transfer, vol. 114, pp. 324-336, 2017.

[15] X. Li, M. Jiang, Z. Huang, and Q. Zhou, "Effect of particle orientation on the drag force in random arrays of prolate ellipsoids in low-Reynolds-number flows," AIChE Journal, vol. 65 , no. 8, 2019.

[16] Q. Zhou and L.-S. Fan, "A second-order accurate immersed boundary-lattice Boltzmann method for particle-laden flows," Journal of Computational Physics, vol. 268, pp. 269-301, 2014.

[17] Z. Huang, C. Zhang, M. Jiang, H. Wang, and Q. Zhou, "Effects of particle velocity fluctuations on inter-phase heat transfer in gas-solid flows," Chemical Engineering Science, vol. 206, pp. 375-386, 2019.

[18] D. P. D. Frank and P. Incropera, Fundamentals of Heat and Mass Transfer, John Wiley \& Sons, Hoboken, NJ, USA, 6th edition, 2007.

[19] Q. Zhou and L.-S. Fan, "Direct numerical simulation of moderate-Reynolds-number flow past arrays of rotating spheres," Physics of Fluids, vol. 27, no. 7, pp. 73306-73423, 2015.

[20] M. A. V. D. Hoef, R. Beetstra, and J. A. M. Kuipers, "LatticeBoltzmann simulations of low-Reynolds-number flow past mono- and bidisperse arrays of spheres: results for the permeability and drag force," Journal of Fluid Mechanics, vol. 528, p. 233, 2005.

[21] W.-P. Breugem, "A second-order accurate immersed boundary method for fully resolved simulations of particleladen flows," Journal of Computational Physics, vol. 231, no. 13, p. 4469, 2012.

[22] B. Sun, S. Tenneti, and S. Subramaniam, "Modeling average gas-solid heat transfer using particle-resolved direct numerical simulation," International Journal of Heat and Mass Transfer, vol. 86, pp. 898-913, 2015.

[23] E. M. Sparrow, J. P. Abraham, and J. C. K. Tong, “Archival correlations for average heat transfer coefficients for noncircular and circular cylinders and for spheres in cross-flow," International Journal of Heat and Mass Transfer, vol. 47, no. 24, pp. 5285-5296, 2004.

[24] Y. A. Cengel, Heat Transfer: A Practical Approach with EES $C D$, p. 896, McGraw-Hill Higher Education, New York, PA, USA, 2002.

[25] Z. Duan, B. He, and Y. Duan, "Sphere drag and heat transfer," Scientific Reports, vol. 5, no. 1, pp. 1-7, 2015. 